\title{
INSURANCE WITH BORROWING: FIRST- AND SECOND-ORDER APPROXIMATIONS
}

\author{
A. A. BOROVKOV, ${ }^{*}$ Sobolev Institute of Mathematics, Novosibirsk
}

\begin{abstract}
We consider the operation of an insurer with a large initial surplus $x>0$. The insurer's surplus process $S(t)$ (with $S(0)=x$ ) evolves in the range $S(t) \geq 0$ as a generalized renewal process with positive mean drift and with jumps at time epochs $T_{1}, T_{2}, \ldots$. At the time $T_{\eta(x)}$ when the process $S(t)$ first becomes negative, the insurer's ruin (in the 'classical' sense) occurs, but the insurer can borrow money via a line of credit. After this moment the process $S(t)$ behaves as a solution to a certain stochastic differential equation which, in general, depends on the indebtedness, $-S(t)$. This behavior of $S(t)$ lasts until the time $\theta(x, y)$ at which the indebtedness reaches some 'critical' level $y>0$. At this moment the line of credit will be closed and the insurer's absolute ruin occurs with deficit $-S(\theta(x, y))$. We find the asymptotics of the absolute ruin probability and the limiting distributions of $\eta(x), \theta(x, y)$, and $-S(\theta(x, y))$ as $x \rightarrow \infty$, assuming that the claim distribution is regularly varying. The second-order approximation to the absolute ruin probability is also obtained. The abovementioned results are obtained by using limiting theorems for the joint distribution of $\eta(x)$ and $-S\left(T_{\eta(x)}\right)$.
\end{abstract}

Keywords: Insurance with borrowing; absolute ruin; deficit at absolute ruin; debit interest; regularly varying distributions; subexponential function; first passage time; excess; compound renewal process; stochastic differential equation; second-order approximation

2000 Mathematics Subject Classification: Primary 60K99; 60B12

\section{Introduction and the main result}

We will begin by describing a general Sparre-Andersen-type risk model (first without any lines of credit). Suppose that claims of positive sizes $\zeta_{n}, n=1,2, \ldots$, are made against the insurer at times

$$
T_{n}:=\sum_{j=1}^{n} \tau_{j}, \quad T_{0}=0,
$$

respectively. We assume that the random vectors $\left(\tau_{j}, \zeta_{j}\right) \stackrel{\mathrm{D}}{=}(\tau, \zeta), j=1,2, \ldots$, are independent, where ' $=$, denotes equality in distribution. The insurance premiums are received continuously at a constant rate $r>0$. Thus, the insurer's surplus at the time $T_{n}$ is

$$
S\left(T_{n}\right)=x-X_{n}, \quad X_{n}:=\sum_{j=1}^{n} \xi_{j}, \quad \xi_{j}:=\zeta_{j}-r \tau_{j} \stackrel{\mathrm{D}}{=} \xi:=\zeta-r \tau .
$$

Received 9 October 2008; revision received 15 June 2009.

* Postal address: Sobolev Institute of Mathematics, Acad. Koptyuga prospect 4, Novosibirsk, 630090, Russia.

Email address: borovkov@math.nsc.ru

Work partially supported by the Russian President's Grant NSh-3695-2008.1 and the RFBR Grant 08-01-00962. 
The sequence $\left\{S\left(T_{n}\right)\right\}$ can be called a discrete-time surplus process. We assume that $\mathrm{E} \xi$ is finite and that

$$
a:=-\mathrm{E} \xi>0 \text {. }
$$

Let $\bar{X}:=\bar{X}_{\infty}$, where $\bar{X}_{n}:=\max _{k \leq n} X_{k}$, and let $x$ be the initial surplus. The event $A(x):=\{\bar{X}>x\}$ means that the insurer's ruin (in the 'classical' sense) takes place. This ruin occurs at the time $T_{\eta(x)}$, where

$$
\eta(x):=\min \left\{k: X_{k}>x\right\}
$$

$\left(\eta(x)=\infty\right.$ if $X_{k} \leq x$ for all $\left.k\right)$.

It is well known (see, e.g. [10]) that if the function

$$
V^{\mathrm{I}}(t):=\int_{t}^{\infty} V(u) \mathrm{d} u, \quad \text { where } \quad V(u):=\mathrm{P}(\xi>u),
$$

is subexponential then

$$
\mathrm{P}(A(x))=\mathrm{P}(\bar{X}>x) \sim \frac{1}{a} V^{\mathrm{I}}(x) \quad \text { as } x \rightarrow \infty,
$$

where the notation $f(x) \sim g(x)$ means that $f(x) / g(x) \rightarrow 1$ as $x \rightarrow \infty$.

If the function $V$ is 'strongly subexponential' (see, e.g. [10]) then

$$
\mathrm{P}(\eta(x) \leq n)=\mathrm{P}\left(\bar{X}_{n}>x\right) \sim \frac{1}{a}\left[V^{\mathrm{I}}(x)-V^{\mathrm{I}}(x+a n)\right] \quad \text { as } x \rightarrow \infty .
$$

If, moreover, $V$ belongs to the class $\mathcal{R}$ of regularly varying functions, that is,

$$
V(t)=t^{-\alpha} L(t), \quad \alpha>1,
$$

where $L(t)$ is a slowly varying at infinity function, then $V^{\mathrm{I}}(x) \sim x V(x) /(\alpha-1)$ as $x \rightarrow \infty$ and

$$
\mathrm{P}\left(\frac{\eta(x)}{x}>u \mid \bar{X}>x\right) \sim(1+a u)^{-\alpha+1}
$$

(see, e.g. [2]).

We can also consider an equivalent model in continuous time. Set

$$
Z_{k}:=\sum_{j=1}^{k} \zeta_{j}, \quad N(t):=\max \left\{k: T_{k} \leq t\right\}, \quad Z(t):=Z_{N(t)} .
$$

Then the insurer's surplus process is

$$
S(t):=x-X(t), \quad X(t):=Z(t)-r t .
$$

In what follows it will be more convenient for us to consider the 'loss process' $X(t)$ (with positive jumps and negative mean drift) as the main subject of study. The value $X(t)$ is the amount of money that the insurer has lost during the time period $(0, t]$. Evidently, $\sup _{t \geq 0} X(t)=$ $\sup _{k \geq 0} X_{k}=\bar{X}$, and so the event of ruin, $A(x)=\{\bar{X}>x\}$, is expressed equally in terms of both processes $X_{k}$ and $X(t)$. The ruin time points for the processes $\left\{X_{k}\right\}$ and $X(t)$ are $\eta(x)$ and $T_{\eta(x)}$, respectively. 
In the present paper we consider the following modification of the above model that assumes that a line of credit will be made available to the insurer when the surplus process drops below 0 . If the event $A(x)$ occurs then, at the time $T_{\eta(x)}$, the insurer borrows an amount of money equal to the deficit at a debit interest force $\delta>0$. Meanwhile, the insurer will repay the debt from his premium income. The loss process $X_{\delta}(t)\left(X_{\delta}(t)=X(t)\right.$ for $\left.t \leq T_{\eta(x)}\right)$ for $t \geq T_{\eta(x)}$ is the solution to the stochastic differential equation

$$
\mathrm{d} X_{\delta}(t)=\left(X_{\delta}(t)-x\right)^{+} \delta \mathrm{d} t+\mathrm{d} Z(t)-r \mathrm{~d} t, \quad X_{\delta}\left(T_{\eta(x)}\right)=X\left(T_{\eta(x)}\right),
$$

where $z^{+}=\max \{0, z\}$. Relation (4) means that the debt is repayed continuously in time proportionally to the current indebtedness $\left(X_{\delta}(t)-x\right)^{+}$.

In [5] such a model was considered, the credit being granted until the current indebtedness $X_{\delta}(t)-x$ reaches the level $r / \delta$ (after this time, $\mathrm{d} X_{\delta}(t) \geq \mathrm{d} Z(t)$ and the path $X_{\delta}(t)$ will grow unboundedly with probability 1 , so the debt can never be repayed). The time

$$
\theta(x, y):=\min \left\{t: X_{\delta}(t)-x>y\right\}
$$

of the first passage of the level $y=r / \delta$ is called, for this model, the absolute ruin time $\left(\theta(x, y)=\infty\right.$ if $X_{\delta}(t)-x \leq y$ for all $\left.t\right)$. At this time the insurer's indebtedness is

$$
D=D\left(x, \frac{r}{\delta}\right):=X_{\delta}\left(\theta\left(x, \frac{r}{\delta}\right)\right)-x \geq \frac{r}{\delta} .
$$

If the absolute ruin does not happen then the process $X_{\delta}(t)$ can return to the range $\left\{X_{\delta} \leq x\right\}$ (when this happens, the debt is repayed) and the insurer will continue to operate in the previous manner; the credit line will again be granted if needed.

There are some other models for insurance with borrowing. They were studied in a number of papers, mostly under the assumptions that $N(t)$ is a Poisson process ( $\tau$ is exponentially distributed), and $\zeta$ and $\tau$ are independent. The absolute ruin probability for exponentially distributed sizes, $\zeta_{j}$, of claims was studied in [7] using a martingale approach. A more general framework was considered in [8] and [9]. The case in which the insurance premiums depend on the surplus $S(t)$ (if $S(t)>0$ ) was considered in, among others, [1], [4], [6], and [11].

The present paper was motivated by [5]. In [5], in particular, the following statement was proved (see [5, Theorem 4.1]).

Theorem 1. ([5].) Let the event $A(x, r / \delta):=\{\theta(x, r / \delta)<\infty\}$ denote absolute ruin. If the function $V^{\mathrm{I}}$ is subexponential then, under some additional assumptions,

$$
\mathrm{P}\left(A\left(x, \frac{r}{\delta}\right)\right) \sim \frac{1}{a} V^{\mathrm{I}}(x) \text { as } x \rightarrow \infty, \text { where } a=-\mathrm{E} \xi=r \mathrm{E} \tau-\mathrm{E} \zeta>0 .
$$

In other words, $\mathrm{P}(A(x, r / \delta)) \sim \mathrm{P}(A(x))$ (see (1)).

Note that if the trajectory of $X(t)$ has reached the level $x+r / \delta$ then the more so the trajectory of the process $X_{\delta}(t)$ (defined on the same probability space) reaches this level because, according to (4), the process $X_{\delta}(t)$ has the same positive jumps as the process $X(t)$ and has less negative drift than $X(t):\left(X_{\delta}(t)-x\right)^{+} \delta-r \geq-r$. Therefore,

$$
A\left(x+\frac{r}{\delta}\right) \subset A\left(x, \frac{r}{\delta}\right) \subset A(x)
$$


and, for $r / \delta=$ constant and subexponential $V^{\mathrm{I}}$,

$$
\frac{V^{\mathrm{I}}(x+r / \delta)}{V^{\mathrm{I}}(x)} \rightarrow 1 \quad \text { as } x \rightarrow \infty .
$$

The last two relations immediately imply that

$$
\mathrm{P}\left(A\left(x, \frac{r}{\delta}\right)\right) \sim \mathrm{P}(A(x)) \sim \mathrm{P}\left(A\left(x+\frac{r}{\delta}\right)\right) \sim \frac{1}{a} V^{\mathrm{I}}(x) .
$$

Thus, (5) is valid without the additional assumptions of Theorem 4.1 of [5], including the requirements that $N(t)$ be a Poisson process, and the components of $\tau$ and $\zeta$ be independent. Therefore, assertion (5) is in fact evident under very wide conditions. Relations (5) and (7) mean that the availability of a line of credit with fixed $\delta$ (or $r / \delta$ ) does not essentially alter the asymptotics of the (absolute) ruin probability. However, we can consider a more general problem that leads to interesting nontrivial results on the asymptotic behavior of the absolute ruin probability, and also that of the joint distribution of the time to the absolute ruin and the deficit at that time. The generalization contains the following elements.

1. As already mentioned, the asymptotics of $\mathrm{P}(A(x, r / \delta))$ in (5) do not depend on $\delta$. The situation may change if $\delta$ is small. This assumption seems natural because the interest force $\delta$ corresponds to a 'small' time unit (say an hour). However, the per cent interest per a long time period $T$ approximately equals $\mathrm{e}^{\delta T}-1$, so if $T$ has the order of $x$ (the amount of debt and the time to repay it under the conditions of Theorem 2, below, have such order) then this per cent interest will be 'reasonable' if the value of $\delta x$ is a 'proper' one (not 'too small' and not 'too large', say less than 1). So we assume that $\delta$ may tend to 0 as $x \rightarrow \infty$, but $\delta x$ remains 'comparable' with 1.

2. To be more precise, we assume that $\delta=\delta\left(x, X_{\delta}(t)-x\right)$ depends also on the value of indebtedness $\left(X_{\delta}(t)-x\right)^{+}$in such a way that in (4) instead of $\left(X_{\delta}(t)-x\right)^{+} \delta$ we can write $p\left(\left(X_{\delta}(t)-x\right) / x\right)$, where $\left(x_{\delta}-x\right) / x$ is the 'normalized' current indebtedness and $p(v)$ is a given fixed function,

$$
p(v)=0 \quad \text { for } v \leq 0 .
$$

If $p(v)=\gamma v^{+}, \gamma=$ constant $>0$, then $\delta$ depends only on $x$ and we have $\delta=\gamma / x$ in (4).

The function $p(v)$ has the following obvious meaning. The amount of interest paid during a small time interval $(t, t+\Delta)$ is $\Delta p(v)$ given that $p(v)$ is continuous and the 'normalized' current indebtedness equals $v$.

The function $p(v)$ in what follows will satisfy some conditions of nondecrease and continuity in a rather weak form (see, e.g. Theorems 2,3 , and 7, below). Thus, we will consider an arbitrary debt repay rule $p(v)$ under condition (8) and certain conditions below.

For the process $X_{p(\cdot)}(t)$ corresponding to an arbitrary function $p(v)$, an analog of (4) can be written in the following form:

$$
\mathrm{d} X_{p(\cdot)}(t)=p\left(\frac{X_{p(\cdot)}(t)-x}{x}\right) \mathrm{d} t-r \mathrm{~d} t+\mathrm{d} Z(t) .
$$

It follows from (9) that $X_{p(\cdot)}(t)$ as a function of $p(\cdot)$ is monotonously increasing: $X_{p(\cdot)}(\cdot) \uparrow$ as $p(\cdot) \uparrow$ (the initial value of $X_{p(\cdot)}(0)=0$ is fixed; $g_{1}(\cdot) \geq g_{2}(\cdot)$ if and only if $g_{1}(t) \geq g_{2}(t)$ for any $t$ ), so $X_{p(\cdot)}(\cdot)$ dominates $X(\cdot)$ since $p(\cdot) \geq 0$. 
3. In the case of the model in (4), the expediency of continuing to credit the insurer is doubtful when the current indebtedness $X_{\delta}(t)-x$ is close to the level $r / \delta$ : in this range the absolute ruin probability is close to 1 . We will assume that, for the general model (9), the line of credit is closed at the time $\theta(x, y)$ when the current indebtedness $X_{p(\cdot)}(t)-x$ exceeds some level $y=\rho x$ for the first time. This level can be chosen to be such that the 'mean local drift' of the process $X_{p(\cdot)}(t)$ in the range $X_{p(\cdot)}(t)<x+y$ remains negative, i.e. (see (9))

$$
r^{*}:=r-p(\rho)>\frac{a_{\zeta}}{a_{\tau}},
$$

where $a_{\zeta}=\mathrm{E} \zeta, a_{\tau}=\mathrm{E} \tau$, and $\rho=y / x$. The inequality in (10) can be rewritten as

$$
a^{*}:=a_{\tau} r^{*}-a_{\zeta}>0
$$

(recall that $a=a_{\tau} r-a_{\zeta}$ ).

For such choice of $y$, the process $X_{p(\cdot)}(t)$ in the range $X_{p(\cdot)}(t) \leq x+y$ is pathwise dominated by the process $X^{*}(t)$, which is defined similarly to $X(t)$ with $r$ replaced by $r^{*}<r$. The process $X^{*}(t)$ still has a negative mean drift like the original process $X(t)$. Also, the chance for the insurer to avoid absolute ruin remains high. According to the notation above, denote by $A(x, y)$ the event of absolute ruin when the line of credit is closed at the deficit level $y$; this occurs at the time $\theta(x, y)$. At this time the insurer's current indebtedness is $X_{p(\cdot)}(\theta(x, y))-x$.

It is clear that, in this new framework, the relations

$$
A(x+y) \subset A(x, y) \subset A(x)
$$

(cf. (6)) and

$$
\mathrm{P}(A(x, y)) \sim \frac{1}{a} V^{\mathrm{I}}(x) \text { for } y \leq c
$$

remain valid for subexponential $V^{\mathrm{I}}$.

4. Now we introduce some restrictions on the distributions of $\xi=\zeta-r \tau$ and $\xi^{*}=\zeta-r^{*} \tau$. As above, let $V(t)=\mathrm{P}(\xi>t)$ and $\mathcal{R}$ be the class of regularly varying functions (see (2)), and let one of the two following alternative conditions hold.

$\left(\mathcal{R}_{1}\right) V \in \mathcal{R}, \alpha \in(1,2)$, the expectation $\mathrm{E} \xi<0$ is finite, and the left distribution tail $\mathrm{P}(\xi<-t)$ of the random variable $\xi$ admits a regularly varying majorant $W$ :

$$
\mathrm{P}(\xi<-t) \leq W(t), \quad W(\cdot) \in \mathcal{R},
$$

such that $W(t)=O(1 / t \ln t)$ as $t \rightarrow \infty$.

$\left(\mathcal{R}_{2}\right) \quad V \in \mathcal{R}, \alpha>2$, and $\mathrm{E} \xi^{2}<\infty$.

The condition that either $\left(\mathcal{R}_{1}\right)$ or $\left(\mathcal{R}_{2}\right)$ holds will be denoted by $(\mathcal{R})$.

Now we can state the first main assertion. It corresponds to the case when the critical level

$$
y=\rho x
$$

is such that the process $X_{p(\cdot)}(t)-x$ attaining this level can still return to the negative range. (Recall that, once the critical level is attained by the process $X_{p(\cdot)}(t)-x$, the line of credit is closed.) 
Theorem 2. Let $a=-\mathrm{E} \xi>0$, let condition $(\mathcal{R})$ hold, and let the process $X_{p(\cdot)}(t)$ be determined by (9) and $X_{p(\cdot)}(0)=0$, where the function $p(u)$ satisfies (8) as well as the condition

$$
\max _{u \leq \rho} p(u)=p(\rho) .
$$

Furthermore, let the level y unboundedly increase so that $\rho=y / x \leq H$ for some (fixed) constant $H$, and let

$$
r^{*}:=r-p(\rho)>\frac{a_{\zeta}}{a_{\tau}} \quad\left(a^{*}:=r^{*} a_{\tau}-a_{\zeta}>0\right)
$$

Then

$$
\mathrm{P}(A(x, y)) \sim \mathrm{P}(A(x+y)) \sim \frac{V^{\mathrm{I}}(x+y)}{a} .
$$

If $y \sim \rho x$ as $x \rightarrow \infty$ and $\rho$ is fixed, then the absolute ruin time $\theta(x, y)$ has the limiting conditional distribution

$$
\lim _{x \rightarrow \infty} \mathrm{P}\left(\frac{\theta(x, y)}{x}>u a_{\tau} \mid A(x, y)\right)=\left(\frac{1+a u+\rho}{1+\rho}\right)^{-\alpha+1} .
$$

The limiting conditional distribution of the deficit $D(x, y):=X_{p(\cdot)}(\theta(x, y))-x$ for $y \sim \rho x$ and $\rho=$ constant is

$$
\lim _{x \rightarrow \infty} \mathrm{P}\left(\frac{D(x, y)}{x}>u \mid A(x, y)\right)=\left(\frac{1+\max (u, \rho)}{1+\rho}\right)^{-\alpha+1} .
$$

We will see later on that Theorem 5 enables us to easily obtain the limiting joint distribution of $\theta(x, y)$ and $D(x, y)$ as well.

Theorem 2 shows that, for the absolute ruin probability to be significantly less than $\mathrm{P}(A(x))$, the parameter $y$ should be chosen as large as possible (within the bounds of (12)). If $y=o(x)$ then introducing the line of credit does not change the asymptotics of the absolute ruin probability $\mathrm{P}(A(x, y))$ in comparison with that for $\mathrm{P}(A(x))$.

Our second main statement corresponds to the case when (12) does not hold and the process $X_{p(\cdot)}(t)-x$, once it has attained the level greater than or equal to $y$, grows unboundedly with high probability, as happens to the solution of (4) for $y=r / \delta=r x / \gamma$ (i.e. for $\rho=r / \gamma$ ).

Theorem 3. Let all the conditions of Theorem 2 except (11) and (12) be satisfied. Instead of (11) and (12) we assume that there exists an $r^{* *}$ such that

$$
r-p(\rho) \leq r^{* *}, \quad a^{* *}:=r^{* *} a_{\tau}-a_{\zeta}<0 .
$$

Moreover, let there exist a unique solution $u=\rho_{0}$ to the equation

$$
r-p(u)-\frac{a_{\zeta}}{a_{\tau}}=0,
$$

and, for each $\varepsilon>0$, there exists $\beta=\beta(\varepsilon)>0$ such that

$$
\min _{u \leq \rho_{0}-\varepsilon} p(u) \leq p\left(\rho_{0}\right)-\beta, \quad \max _{u \geq \rho_{0}+\varepsilon} p(u) \geq p\left(\rho_{0}\right)+\beta
$$

(this implies that $\rho_{0}<\rho$ ). Then, for $y_{0}=\rho_{0} x<y$,

$$
\mathrm{P}(A(x, y)) \sim \frac{V^{\mathrm{I}}\left(x+y_{0}\right)}{a} .
$$


The level $y_{0}=\rho_{0} x$ is such that the mean drift of the process $X_{p(\cdot)}(t)$ in the range of its values close to $y_{0}$ is close to 0 .

Under the conditions of Theorem 3, we can also obtain the limiting distributions of $\theta(x, y)$ and $D(x, y)$, as was the case in Theorem 2 (see the remarks after the proof of Theorem 5, below). But comparison of statements (13) and (19) shows that raising the 'critical' level $y$ above $y_{0}$ does not reduce the absolute ruin probability. This is why it is inexpedient to choose $y>y_{0}$ and, hence, studying the limiting distributions of $\theta(x, y)$ and $D(x, y)$ is not so interesting for such $y$.

The aforesaid means that there exists a lower asymptotic bound for the absolute ruin probability (see also the proofs of Theorems 2 and 3 in Section 3).

Corollary 1. Let all the conditions of Theorem 2 except (11) and(12) be satisfied. Furthermore, let there exist a unique solution $u=\rho_{0}$ to (17), and let (18) hold. Then, for $x \rightarrow \infty$ and all $y$,

$$
\mathrm{P}(A(x, y)) \geq \frac{1}{a} V^{\mathrm{I}}\left(x\left(1+\rho_{0}\right)\right)(1+o(1)) .
$$

The right-hand side of the last relation can be replaced with $\mathrm{P}\left(A\left(x, y_{0}\right)\right)(1+o(1))$, where $y_{0}=\rho_{0} x$.

If $p(u)=\gamma u^{+}$then

$$
\rho_{0}=\frac{1}{\gamma}\left(r-\frac{a_{\zeta}}{a_{\tau}}\right) .
$$

The proofs of Theorems 2 and 3 are presented in Section 3. A theorem on the asymptotics of the joint distribution of $\eta(x)$ and $\chi(x):=X_{\eta(x)}-x$ will be stated and proved in Section 2; this theorem will be needed is Section 3. In Section 4 a 'conditional' law of large numbers is proved for the process describing the evolution of the indebtedness in the presence of a credit line. In Section 5 integro-local limiting theorems for the joint distribution of $\eta(x)$ and $\chi(x)$ are obtained. Based on these theorems the second-order approximation to the absolute ruin probability $\mathrm{P}(A(x, y))$ is found in Section 6 .

\section{The asymptotics of the joint distribution of $\eta(x)$ and $\chi(x)=X_{\eta(x)}-x$}

In this section we use the notation of Section 1.

Theorem 4. Let $a=-\mathrm{E} \xi>0, h>0$, and $N>0$ be arbitrary fixed numbers. Furthermore, let condition $(\mathcal{R})$ be satisfied. Then, for all

$$
u \geq(x+a n) h
$$

the asymptotic representation

$$
\mathrm{P}(\eta(x)=n, \chi(x)>u) \sim V(x+a n+u)
$$

as $x \rightarrow \infty$ holds uniformly in all $n \leq N x$.

Theorem 4 implies the following result (in addition to (3)). 
Corollary 2. Let all the conditions of Theorem 4 except (20) be satisfied. Then, for $n \leq N x$, $u=z x$, and each fixed $N>0$ and $z>0$, the relations

$$
\begin{gathered}
\mathrm{P}(\infty>\eta(x) \geq n, \chi(x)>u) \sim \frac{1}{a} V^{\mathrm{I}}(x+a n+z x), \\
\mathrm{P}(\bar{X}>x, \chi(x)>u) \sim \frac{1}{a} V^{\mathrm{I}}(x(1+z)), \\
\mathrm{P}\left(\frac{\chi(x)}{x}>z \mid \bar{X}>x\right) \sim(1+z)^{-\alpha+1}
\end{gathered}
$$

hold as $x \rightarrow \infty$. Moreover, relation (23) remains valid for $u=o(x)$.

Note that, by (23),

$$
\mathrm{P}(\bar{X}>x, \chi(x)>u) \sim \frac{1}{a} V^{\mathrm{I}}(x+u) \sim \mathrm{P}(\bar{X}>x+u) .
$$

Proof of Theorem 4. Set $\xi_{j}^{0}:=\xi_{j}+a, X_{n}^{0}:=X_{n}+a n, V^{0}(t)=\mathrm{P}\left(\xi^{0}>t\right) \sim V(t)$ as $t \rightarrow \infty$, and $G_{n}:=\left\{\bar{X}_{n-1}<x\right\}$.

First consider the case when condition $\left(\mathcal{R}_{2}\right)$ holds. Let $\varepsilon=\varepsilon(n)$ tend to 0 so slowly that $\varepsilon n \gg \sqrt{n \ln n}$ as $n \rightarrow \infty$. Then $\mathrm{P}\left(\left|X_{n-1}^{0}\right|>(x+a n)(1+h) \varepsilon\right) \rightarrow 0$ and $\mathrm{P}\left(G_{n}\right) \rightarrow 1$ as $x \rightarrow \infty$ uniformly in $n$ (see, e.g. Corollary 4.1 .9 of [3]). Furthermore, for $u=(x+a n) z$ and $z \geq h$, we have

$$
\begin{aligned}
\mathrm{P}(\eta(x)=n, \chi(x)>u) & =\mathrm{P}\left(\bar{X}_{n-1}<x, X_{n-1}^{0}+\xi_{n}^{0}>(x+a n)(1+z)\right) \\
& =\mathrm{E}\left[V^{0}\left((x+a n)(1+z)-X_{n-1}^{0}\right) ; G_{n}\right] \\
& =E_{1}+E_{2}+E_{3}+E_{4},
\end{aligned}
$$

where, for $w:=(x+a n)(1+z)=x+a n+u$,

$$
\begin{aligned}
E_{1} & :=\mathrm{E}\left[V^{0}\left(w-X_{n-1}^{0}\right) ; G_{n},\left|X_{n-1}^{0}\right|<\varepsilon w\right] \sim V^{0}(w), \\
E_{2} & :=\mathrm{E}\left[V^{0}\left(w-X_{n-1}^{0}\right) ; G_{n}, X_{n-1}^{0}<-\varepsilon w\right]=V^{0}(w) o(1), \\
E_{3} & :=\mathrm{E}\left[V^{0}\left(w-X_{n-1}^{0}\right) ; G_{n}, X_{n-1}^{0} \in\left[\varepsilon w, \frac{x+a n}{2}\right]\right]=V^{0}\left(\frac{x+a n}{2}\right) o(1)=V^{0}(w) o(1), \\
E_{4} & :=\mathrm{E}\left[V^{0}\left(w-X_{n-1}^{0}\right) ; G_{n}, X_{n-1}^{0} \in\left(\frac{x+a n}{2}, x+a n\right)\right] \\
& \leq V^{0}(z(x+a n)) O\left(n V^{0}(x+a n)\right) \\
& =V^{0}(w) o(1),
\end{aligned}
$$

as $x \rightarrow \infty$ (see, e.g. Theorem 3.4.1 of [3]). Evidently, all the $O(\cdot)$ and $o(\cdot)$ terms here are uniform in $n$ as $x \rightarrow \infty$. Adding up the above relations for $E_{1}-E_{4}$ we obtain

$$
\mathrm{P}(\eta(x)=n, \chi(x)>u) \sim V^{0}((x+a n)(1+z)) \sim V(x+a n+u) .
$$

This implies (21).

In the case when $\left(\mathcal{R}_{1}\right)$ holds the above argument remains valid as well. The only thing to be changed is the choice of the sequence $\varepsilon=\varepsilon(n)$. This sequence is to be chosen such that $\varepsilon n \gg \sigma(n)$, where $\sigma(n)=V^{(-1)}(1 / n)$ and $V^{(-1)}$ is the generalized inverse of $V$ (i.e. the corresponding quantile transform). Then $\mathrm{P}\left(\left|X_{n-1}^{0}\right|>(x+a n)(1+z) \varepsilon\right) \rightarrow 0$ as $x \rightarrow \infty$ uniformly in $n$ (see, e.g. Corollary 3.1.3 of [3]). This completes the proof. 
Proof of Corollary 2. If $u=z x$ for some fixed $z>0$ then, for $n \leq N x$ (with some fixed $N>0)$, the relations

$$
u \geq \max \left(z x, \frac{z a n}{a N}\right) \geq(x+a n) z h
$$

are valid for some $h>0$. These relations imply (20). Hence, (21) is valid. Thus,

$$
\mathrm{P}(\infty>\eta(x) \geq n, \chi(x)>u) \sim \sum_{k=n}^{\lfloor N x\rfloor} V(x+a k+z x)+O(\mathrm{P}(\eta(x)>N x)) .
$$

The first term on the right-hand side of (25) can be made arbitrarily close (in the sense of the asymptotic equivalence) to $(1 / a) V^{\mathrm{I}}(x+a n+z x)$ by choosing a suitable $N$. By virtue of (3), choosing an appropriate $N$, we can make the second term on the right-hand side of (25) arbitrarily small compared to $V^{\mathrm{I}}(x)$. This proves (22). Relation (23) follows from (22) for $n=1$. Relation (24) follows from (1) and (23).

Since (23) is also valid for $u=0$ in view of (1), relation (23) for $u=o(x)$ easily follows from considerations of monotonicity and the relation $V^{\mathrm{I}}(x+o(x)) \sim V^{\mathrm{I}}(x)$. This completes the proof.

The assertion of Theorem 4 is obtained under the restriction $u \geq(x+a n) h, h>0$. Studying the asymptotics of the left-hand side of (21) for $u=o(x+a n)$ requires employing more refined approaches dealing with integro-local theorems for $X_{n}$. The same can be said about relation (23) for $u \gg x$.

\section{Proofs of Theorems 2 and 3}

In this section we return to the model formulated in Section 1.

Proof of Theorem 2. First we prove (13). For bounded $y$, the proof of relation (13) is completely analogous to the proof of (7). Thus, in the sequel we will assume that $y \rightarrow \infty$ as $x \rightarrow \infty$. Represent $\mathrm{P}(A(x, y))$ as

$$
\mathrm{P}(A(x, y))=P_{1}+P_{2}+P_{3},
$$

where

$$
P_{1}:=\sum_{k=1}^{\infty} \mathrm{P}(\eta(x)=k, \chi(x)>y)=\mathrm{P}(\bar{X}>x, \chi(x)>y) \sim \frac{1}{a} V^{\mathrm{I}}(x+y)
$$

by Corollary 2. Furthermore, consider, for $\varepsilon \in(0,1)$,

$$
P_{2}:=\int_{0}^{y-\varepsilon y} \mathrm{P}(\eta(x)<\infty, \chi(x) \in \mathrm{d} v) \mathrm{P}\left(\sup _{u \geq 0} X_{p(\cdot), v}(u)>y-v\right),
$$

where

$$
X_{p(\cdot), v}(u):=X_{p(\cdot)}\left(T_{\eta(x)}+u\right)-v, \quad v=X\left(T_{\eta(x)}\right), u \geq 0 .
$$

The random variables $\eta(x)$ and $T_{\eta(x)}$ are stopping times for the processes $X_{k}$ and $X(t)$, respectively. Hence, the process $X_{p(\cdot), v}(u)$ depends on $v$ only (for $\chi(x)=v$ ) and does not 
depend on $X_{p(\cdot)}(t)$ for $t \leq T_{\eta(x)}$, and, moreover, is dominated in the range of values $(-\infty, y-v)$ by the process distributed equally with the process $X^{*}(u)$, so that

$$
\mathrm{P}\left(\sup _{u \geq 0} X_{p(\cdot), v}(u)>y-v\right) \leq \mathrm{P}\left(\sup _{u \geq 0} X^{*}(u)>y-v\right) .
$$

Since $y-v \geq \varepsilon y$ in the integration domain in (28), we see that, for $X^{*}:=\sup _{u \geq 0} X^{*}(u)$,

$$
\mathrm{P}\left(\sup _{u \geq 0} X_{p(\cdot), v}(u)>y-v\right) \leq \mathrm{P}\left(X^{*}>\varepsilon y\right) \rightarrow 0,
$$

if $\varepsilon=\varepsilon(y) \rightarrow 0$ is chosen so that $\varepsilon y \rightarrow \infty$ as $y \rightarrow \infty$. Thus,

$$
P_{2} \leq \mathrm{P}(\bar{X}>x) o(1) \text {. }
$$

Furthermore, we have

$$
\begin{aligned}
P_{3} & :=\int_{y-\varepsilon y}^{y} \mathrm{P}(\eta(x)<\infty, \chi(x) \in \mathrm{d} v) \mathrm{P}\left(\sup _{u \geq 0} X_{p(\cdot), v}>y-v\right) \\
& \leq \mathrm{P}(\bar{X}>x, \chi(x) \in[y-\varepsilon y, y]),
\end{aligned}
$$

where $\varepsilon y=o(x)$ and the right-hand side of (29) is $o\left(V^{\mathrm{I}}(x)\right)$ by Corollary 2. As a result, we obtain

$$
P_{2}+P_{3}=o\left(V^{\mathrm{I}}(x)\right) .
$$

Since $V^{\mathrm{I}}(x)=O\left(V^{\mathrm{I}}(x+y)\right)$ for $y \leq H x$, combining (26), (27), and (30) proves relation (13).

The aforesaid means that the event $A(x, y)$ can be represented as

$$
A(x, y)=A(x) \cap[B \cup C],
$$

where $B=\{\chi(x)>y\}$ and $B \cap C=\varnothing$, and, for $y \rightarrow \infty$, the relation $\mathrm{P}(A(x) C)=$ $o(\mathrm{P}(A(x) B))$ is valid. Therefore, for $y=\rho x$,

$$
\begin{aligned}
\mathrm{P}(\infty>\theta(x, y) \geq v x \mid A(x, y)) & =\frac{\mathrm{P}(\infty>\theta(x, y) \geq v x ; A(x) B)}{\mathrm{P}(A(x) B)(1+o(1))}+o(1) \\
& =\frac{\mathrm{P}\left(\infty>T_{\eta(x)} \geq v x ; \chi(x)>\rho x \mid A(x)\right)}{\mathrm{P}(\chi(x)>\rho x \mid A(x))}+o(1),
\end{aligned}
$$

where

$$
\frac{T_{\eta(x)}}{x}=\frac{T_{\eta(x)}}{\eta(x)} \frac{\eta(x)}{x}, \quad \frac{T_{\eta(x)}}{\eta(x)} \longrightarrow a_{\tau} \quad \text { almost surely }
$$

as $x \rightarrow \infty$. Hence, by Corollary 2 ,

$$
\mathrm{P}\left(\infty>\frac{\theta(x, y)}{x} \geq v a_{\tau} \mid A(x, y)\right) \sim \frac{V^{\mathrm{I}}(x(1+a v+\rho))}{V^{\mathrm{I}}(x(1+\rho))} \sim\left(\frac{1+a v+\rho}{1+\rho}\right)^{-\alpha+1} .
$$

This proves (14).

Evidently, the limiting conditional distribution of the indebtedness $D(x, y)$ given the event $A(x, y)$ coincides with the limiting conditional distribution of $\chi(x)$ given that $\bar{X}>x$ and $\chi(x)>y$. Since, for any events $A, B$, and $C$,

$$
\mathrm{P}(A \mid B C)=\frac{\mathrm{P}(A B C)}{\mathrm{P}(B C)}=\frac{\mathrm{P}(A B C)}{\mathrm{P}(C)} \frac{\mathrm{P}(C)}{\mathrm{P}(B C)}=\frac{\mathrm{P}(A B \mid C)}{\mathrm{P}(B \mid C)},
$$


we have, for $y=\rho x$ and $\rho=$ constant,

$$
\begin{aligned}
& \mathrm{P}\left(\frac{\chi(x)}{x}>v \mid \bar{X}>x, \chi(x)>y\right) \\
& \quad=\mathrm{P}\left(\frac{\chi(x)}{x}>v, \frac{\chi(x)}{x}>\rho \mid \bar{X}>x\right) / \mathrm{P}\left(\frac{\chi(x)}{x}>\rho \mid \bar{X}>x\right) .
\end{aligned}
$$

By Corollary 2, this yields

$$
\lim _{x \rightarrow \infty} \mathrm{P}\left(\frac{D(x, y)}{x}>v \mid A(x, y)\right)=\left(\frac{1+\max (v, \rho)}{1+\rho}\right)^{-\alpha+1} .
$$

This proves (15), completing the proof.

Proof of Theorem 3. The argument below does not differ significantly from the previous proof. Instead of (26) we now represent $\mathrm{P}(A(x, y))$ as

$$
\mathrm{P}(A(x, y))=P_{1}+P_{2}+P_{3}+P_{4},
$$

where $P_{1}:=\mathrm{P}(\bar{X}>x, \chi(x) \geq y)$ coincides with (27). Furthermore, by analogy with (28) we have

$$
P_{2}:=\sum_{k=1}^{\infty} \int_{0}^{y_{0}(1-\varepsilon)} \mathrm{P}(\eta(x)=k, \chi(x) \in \mathrm{d} v) \mathrm{P}\left(\sup _{u \geq 0} X_{p(\cdot), v}(u) \geq y-v\right)=o\left(V^{\mathrm{I}}(x)\right) .
$$

The probabilities $P_{3}$ and $P_{4}$ have the same form but with the integrals taken over the intervals $\left(y_{0}(1-\varepsilon), y_{0}(1+\varepsilon)\right)$ and $\left(y_{0}(1+\varepsilon), y\right)$, respectively. It is clear that, by Corollary 2 , the value of $P_{3}$ can be made arbitrarily small compared to $V^{\mathrm{I}}(x)$ by choosing a sufficiently small $\varepsilon>0$.

Now consider $P_{4}$. The process $X_{p(\cdot), v}(t)$ in the range of values $\left(y_{0}(1+\varepsilon), y\right)$ is minorized by the process $X^{* *}(t)$ of the form similar to $X^{*}(t)$ but with positive mean drift. Hence,

$$
\begin{gathered}
\mathrm{P}\left(\sup _{v \geq 0} X_{p(\cdot), v}(v) \geq y-v\right) \geq \mathrm{P}\left(\sup _{v \geq 0} X^{* *}(v) \geq y-v\right) \rightarrow 1, \\
P_{4} \sim \mathrm{P}\left(\bar{X}>x, \chi(x) \in\left[y_{0}(1+\varepsilon), y\right]\right) .
\end{gathered}
$$

Therefore,

$$
P_{1}+P_{4} \sim \mathrm{P}\left(\bar{X}>x, \chi(x)>y_{0}(1+\varepsilon)\right) .
$$

The ratio of the right-hand side of the last relation to the value $(1 / a) V^{\mathrm{I}}\left(x+y_{0}\right)$ can be made arbitrarily close to 1 by choosing an appropriate $\varepsilon$. This completes the proof.

\section{4. 'Conditional' law of large numbers for the time to repay the debt}

Consider the indebtedness process

$$
Y(t)=X_{p(\cdot)}\left(T_{\eta(x)}+t\right)-x
$$

under the conditions of Theorem 2. We assume that the initial value (for $t=0$ ) of the process lies in $(0, y)$. Using the methods and terminology of queueing theory, we will consider the 
so-called fluid limit. To do this, we scale both the time and the amount of money by dividing these quantities by $x$. In other words, we consider the process

$$
d_{x}(t):=\frac{1}{x} Y(x t)
$$

and study its limiting distribution as $x \rightarrow \infty$ under the assumption that the initial (random) value of $d_{x}(0) \in(0, \rho)$ is fixed.

Theorem 5. Let $p(v)$ in (9) be a continuous function on $[0, \rho]$ satisfying conditions (8), (11), and (12). Let $d(t)$ be the solution to the equation

$$
d^{\prime}(t)=p(d(t))-b, \quad d(0)=u \in(0, \rho),
$$

where $b=r-a_{\zeta} / a_{\tau}>0$. Then the function $d(t)$ is strictly decreasing and there exists $a$ unique solution $t_{0}$ to the equation $d(t)=0$.

Furthermore, let the conditions of Theorem 2 be satisfied and let $d_{x}(0) \in(u-\varepsilon(x), u+\varepsilon(x))$, $\varepsilon(x) \rightarrow \infty$ as $x \rightarrow \infty$. Then, for each fixed $T>0$, the following convergence of the process $d_{x}(t)$ to the function $d(t)$ takes place:

$$
\sup _{0 \leq t \leq T}\left|d_{x}(t)-d(t)\right| \stackrel{\mathrm{P}}{\rightarrow} 0 \quad \text { as } x \rightarrow \infty
$$

where $\stackrel{\text { P }}{\rightarrow}$ ' denotes convergence in probability.

In particular, if $p(v)=\gamma v^{+}$then the solutions $d(t)$ and $t_{0}$ are

$$
\begin{gathered}
d(t)=\gamma^{-1}\left[b+(\gamma u-b) \mathrm{e}^{\gamma t}\right], \\
t_{0}=\gamma^{-1} \ln \frac{b}{b-\gamma u} .
\end{gathered}
$$

Therefore, for $p(v)=\gamma v^{+}$and given the event $A(x) \bar{A}(x, y)$, the time to repay the debt behaves asymptotically as

$$
\frac{x}{\gamma} \ln \frac{b}{\gamma-p \psi},
$$

where the random variable $\psi$ has the distribution

$$
\mathrm{P}(\psi<v)=\frac{1-(1+v)^{-\alpha+1}}{1-(1+\rho)^{-\alpha+1}}, \quad v \in[0, \rho]
$$

(see (24)).

Proof. We will only sketch the proof as all the arguments involved are quite standard.

We will assume that the process $Y(t), t \geq 0$, is driven by a new sequence $\left\{\left(\tau_{j}, \zeta_{j}\right)\right\}$ (or a new process $Z(t))$ which does not depend on the sequence driving the process $X_{p(\cdot)}(t)$ up to the time $T_{\eta(x)}$. From now on, almost-sure convergence will be considered with respect to the distribution of this new sequence. The new process $Z(t)$ driving $Y(t)$ for $t \geq 0$, by virtue of the strong law of large numbers, possesses the following property: for each fixed $T$ and $\varepsilon>0$, we have

$$
\mathrm{P}\left(\sup _{t_{0} \leq t \leq T x}\left|Z(t)-\frac{t a_{\zeta}}{a_{\tau}}\right|>\varepsilon x\right) \rightarrow 0
$$


as $t_{0} \rightarrow \infty$ and $x \rightarrow \infty$. Hence, all the increments of the process $Z(\cdot)$ on the time intervals $(x t, x t(1+\Delta))$ for all large enough $x$ lie in the interval

$$
\left[\frac{x \Delta a_{\zeta}}{a_{\tau}}-2 \varepsilon x, \frac{x \Delta a_{\zeta}}{a_{\tau}}+2 \varepsilon x\right]
$$

with high probability. This statement remains valid when $\varepsilon=\varepsilon(x)$ tends to 0 (as $x \rightarrow \infty$ ) slowly enough.

We have $p(v) \leq p(\rho)$ for $v \leq \rho$. Hence, for $d_{x}(t) \in(0, \rho)$, the increments of the process $d_{x}(t)$ on $(t, t+\Delta)$ (which are determined by the increments of the process $X_{p(\cdot)}(t)$ in (9)) for $\Delta>\varepsilon$ lie within the bounds

$$
\Delta\left[p\left(d_{x}(t)\right) \pm \omega_{\Delta}-r+\frac{a_{\zeta}}{a_{\tau}}(1 \pm \varepsilon)\right],
$$

where $\omega_{\Delta}=\sup _{v \leq \rho}|p(v+\Delta)-p(v)|$ is the modulus of continuity of the function $p(\cdot)$.

In view of the aforesaid and (9), the relation

$$
\sup _{t \leq T}\left|\frac{d_{x}(t+\Delta)-d_{x}(t)}{\Delta}-p\left(d_{x}(t)\right)+r-\frac{a_{\zeta}}{a_{\tau}}\right| \stackrel{\mathrm{P}}{\rightarrow} 0
$$

holds as $\Delta \rightarrow 0, \Delta x \rightarrow \infty$, and $\Delta \gg \varepsilon(x)$. These relations imply that the values of $d_{x}(t+\Delta)$ are determined (up to $o_{p}(\Delta)$ ) by the value of $d_{x}(t)$. Hence, for $t \leq T$, the values $d_{x}(t)$ are determined by $d_{x}(0)$ up to $o_{p}(1)$.

Because $\left|d^{\prime}(t)\right|$ is bounded, (31) can be dealt with in a similar way. Equation (31) implies that

$$
\sup _{t \leq T}\left|\frac{d(t+\Delta)-d(t)}{\Delta}-p(d(t))+b\right| \leq p \omega_{\Delta} \rightarrow 0
$$

as $\Delta \rightarrow 0$. Hence, $d(t+\Delta)$ is determined by $d(t)$ up to $\Delta \omega_{\Delta}$ uniformly in $t \leq T$. In (35) we have $r-a_{\zeta} / a_{\tau}=b$. Thus, we see that, given the initial values $d(0)=u$ and $d_{x}(0) \in(u-\varepsilon(x), u+\varepsilon(x))$, the functions $d(t)$ and $d_{x}(t)$ are constructed using identical procedures and from 'elements' which evidently yield the same values (up to $o_{p}(1)$ ) along the paths of these processes. This proves (32).

That $d(t)$ is strictly decreasing follows from the relations

$$
d^{\prime}(t)=p(d(t))-b<p(\rho)-b<0 .
$$

If $p(v)=\gamma v^{+}$then (31) turns into the equation

$$
d^{\prime}(t)=-b+\gamma d(t)
$$

where $-b+\gamma \rho<0$ (see (10)). After changing the variable

$$
f(t)=\gamma d(t)-b
$$

we obtain

$$
f^{\prime}(t)=\gamma f(t), \quad f(t)=c \mathrm{e}^{\gamma t}, \quad d(t)=\frac{b+f(t)}{\gamma}=\frac{b+c \mathrm{e}^{\gamma t}}{\gamma} .
$$

Since $d(0)=u<\rho$, we have $c=u \gamma-b<\rho \gamma-b<0$, which proves (33). Solving the equation $d(t)=0$ we obtain (34). This completes the proof of the theorem. 
Based on the argument above, we can now describe the limiting distribution of the time to absolute ruin $\theta(x, y)$ and the amount of indebtedness $X_{p(\cdot)}(\theta(x, y))-x$ under the conditions of Theorem 3. As seen above, under these conditions, the event $A(x, y)$, roughly speaking, almost coincides with the event $\left\{\chi(x)>y_{0}\right\}$. Besides, if $\chi(x)>y$ then $\theta(x, y)=T_{\eta(x)}$. If $\chi(x) \in\left(y_{0}, y\right)$ then $\theta(x, y)=T_{\eta(x)}+t_{\rho} x\left(1+o_{p}(1)\right)$, where $t_{\rho}$ is the solution to the equation $d(t)=\rho$ and $d(t)$ is the solution to the differential equation (31) under the random initial condition $d(0)=u=\chi(x) / x \in\left(\rho_{0}, \rho\right)\left(d^{\prime}(t)>0\right.$ if $u \in\left(\rho_{0}, \rho\right)$ under the conditions of Theorem 3). Accordingly, the indebtedness coincides with $\chi(x)$ if $\chi(x)>y$, and equals $y$ if $\chi(x) \in\left(y_{0}, y\right)$. From this we can easily obtain the limiting distributions for $\theta(x, y)$ and the indebtedness.

\section{Integro-local theorems for the joint distribution of $\eta(x)$ and $\chi(x)$}

Our next goal is to refine statement (13) of Theorem 2 and to find the second-order asymptotics (i.e. two-term asymptotic expansion) for $\mathrm{P}(A(x, y))$. In order to do this, we need an integro-local theorem for the joint distribution of $\eta(x)$ and $\chi(x)$ as $x \rightarrow \infty$, which refines Theorem 4. For the sake of simplicity, we restrict ourselves to studying only the case when condition $\left(\mathcal{R}_{2}\right)$ holds. Denote by $\Delta[x)$ the interval

$$
\Delta[x):=[x, x+\Delta), \quad \Delta>0 .
$$

We also assume that the following condition holds.

$\left(\mathcal{R}_{3}\right)$ Condition $\left(\mathcal{R}_{2}\right)$ is satisfied, and, for each fixed $\Delta>0$,

$$
\mathrm{P}(\xi \in \Delta[x))=\Delta v(x)(1+o(1))
$$

as $x \rightarrow \infty$, where $v(x):=\alpha x^{-\alpha-1} L(x)$ and $L$ is the slowly varying at infinity function from (2).

This condition holds whenever condition $\left(\mathcal{R}_{2}\right)$ is satisfied and the function $L$ in $(2)$ is differentiable with

$$
L^{\prime}(x)=o\left(\frac{L(x)}{x}\right) \quad \text { as } x \rightarrow \infty .
$$

In this case $v(x) \sim V^{\prime}(x)$ as $x \rightarrow \infty$.

The following integro-local theorem holds.

Theorem 6. Let $\mathrm{E} \xi=-a<0$, and let condition $\left(\mathcal{R}_{3}\right)$ be satisfied. Then

$$
\begin{gathered}
\mathrm{P}(\eta(x)=n, \chi(x) \in \Delta[y))=\Delta\left[v(x+y+a n)(1+o(1))+r_{1}\right], \\
\mathrm{P}(\chi(x) \in \Delta[y), \eta(x)<\infty)=\frac{\Delta}{a}\left[V(x+y)(1+o(1))+r_{2}\right],
\end{gathered}
$$

as $x \rightarrow \infty$ and $y \rightarrow \infty$, where

$$
\begin{gathered}
\left|r_{1}\right| \leq n v\left(\frac{x+a n}{2}\right) V(y)(1+o(1)), \\
\left|r_{2}\right| \leq \frac{2}{a} V^{\mathrm{I}}\left(\frac{x}{2}\right) V(y)(1+o(1)) .
\end{gathered}
$$

The o(1) remainder terms in (36) and (38) are uniform in $n$. 
Relations (36) and (37) obviously imply the following corollary.

Corollary 3. If the conditions of Theorem 6 are satisfied, $y \rightarrow \infty, y<c x$ for some $c<\infty$, and

$$
n V(y) \rightarrow 0
$$

then

$$
\mathrm{P}(\eta(x)=n, \chi(x) \in \Delta[y)) \sim \Delta v(x+y+a n) .
$$

If, instead of (39), the relation

$$
x V(y) \rightarrow 0
$$

holds then

$$
\mathrm{P}(\chi(x) \in \Delta[y), \eta(x)<\infty) \sim \frac{\Delta}{a} V(x+y) .
$$

Conditions (39) and (40) surely hold if $n, x$, and $y$ grow at the same rate. But if, say, $n V(y) \rightarrow \infty$ then representation (36) becomes just a bound. This is due to crudeness of the estimates used in the proof of the theorem.

Proof of Theorem 6. For the sake of convenience, we will consider the following expression instead of the left-hand side of (36):

$$
\begin{aligned}
\mathrm{P}(\eta(x)=n+1, \chi(x) \in \Delta[y)) & =\int_{-\infty}^{x} \mathrm{P}\left(\bar{X}_{n}<x, X_{n} \in \mathrm{d} u\right) \mathrm{P}\left(\xi_{n+1} \in x-u+\Delta[y)\right) \\
& =\Delta \int_{-\infty}^{x} \mathrm{P}\left(\bar{X}_{n}<x, X_{n} \in \mathrm{d} u\right) v(x-u+y)(1+o(1)) .
\end{aligned}
$$

Set

$$
b_{x, n}:=\max (\sqrt{x} \ln x, \sqrt{n} \ln n), \quad P_{n}(\mathrm{~d} u):=\mathrm{P}\left(\bar{X}_{n}<x, X \in \mathrm{d} u\right) v(x-u+y) .
$$

We have

$$
\mathrm{P}\left(\bar{X}_{n}<x, X_{n} \in\left(-a n-b_{x, n},-a n+b_{x, n}\right)\right) \rightarrow 1
$$

as $x \rightarrow \infty$. Therefore, the main part in (41) equals

$$
\int_{-a n-b_{x, n}}^{-a n+b_{x, n}} P_{n}(\mathrm{~d} u) \sim \Delta v(x+y+a n) .
$$

Now we will bound the other parts of the integral in (41). Since $v(x)$ is 'asymptotically' nonincreasing, the following relation holds:

$$
\int_{-\infty}^{-a n-b_{x, n}} P_{n}(\mathrm{~d} u) \leq \Delta v(x+y+a n) o(1) .
$$

Now consider the integral $\int_{-a n+b_{x, n}}^{x} P_{n}(\mathrm{~d} u)$ in (41). Using the notation given in the proof of Theorem 4, we obtain

$$
\begin{aligned}
\int_{-a n+b_{x, n}}^{x} P_{n}(\mathrm{~d} u) & \leq \int_{b_{x, n}}^{x+a n} \mathrm{P}\left(X_{n}^{0} \in \mathrm{d} u\right) v(x+y+a n-u) \\
& =\int_{b_{x, n}}^{(x+a n) / 2} P_{n}(\mathrm{~d} u)+\int_{(x+a n) / 2}^{x+a n} P_{n}(\mathrm{~d} u) .
\end{aligned}
$$


The first integral on the right-hand side admits the following upper bound:

$$
v\left(\frac{x+a n}{2}+y\right)(1+o(1)) \mathrm{P}\left(X_{n}^{0}>b_{x, n}\right)=v\left(\frac{x+a n}{2}+y\right) o(1) .
$$

The second integral on the right-hand side of (44), by virtue of condition $\left(\mathcal{R}_{3}\right)$ and the integrolocal theorem for $X_{n}^{0}$ (see Theorem 4.7.1 of [3]), can be bounded by the sum

$$
n \sum_{k \in I} v(k) v(x+y+a n-k)(1+o(1)) \leq n v\left(\frac{x+a n}{2}\right) V(y)(1+o(1)),
$$

where $I$ denotes the set of all integers from the interval $((x+a n) / 2, x+a n)$. Thus, we have the following bound for the integral on the left-hand side of (44):

$$
o(v(x+y+a n))+n v\left(\frac{x+a n}{2}\right) V(y)(1+o(1)) .
$$

Combining this with relations (42) and (43), we obtain the first assertion of the theorem.

To prove the second assertion of the theorem, we sum up the right-hand sides of (36) for $n$ running from 1 to $\infty$. Summing up the main parts yields $(\Delta / a) V(x+y)(1+o(1))$. Summing up the remainder terms $r_{1}$ yields the sum

$$
\sum_{n=1}^{\infty} n v\left(\frac{x+a n}{2}\right)
$$

It is easy to see that this sum is asymptotically equivalent to $(2 / a) V^{\mathrm{I}}(x / 2)$. Therefore, $\left|r_{2}\right| \leq$ $(2 / a) V^{\mathrm{I}}(x / 2) V(y)(1+o(1))$. This completes the proof of the theorem.

\section{Second-order approximation for the absolute ruin probability}

The accuracy of the approximation to the absolute ruin probability $\mathrm{P}(A(x, y))$ is of crucial importance for applications. The accuracy of approximation (13) in Theorem 2 is not always satisfactory. In this section we establish the following assertion.

Theorem 7. Let the conditions of Theorem 2 and condition $\left(\mathcal{R}_{3}\right)$ be satisfied. Moreover, let the function $p(v)$ be continuous and nondecreasing in a left half-neighborhood of the point $\rho=y / x$. Then, for $y=\rho x$ and $\rho \in\left(0, \rho_{0}\right)$,

$$
\mathrm{P}(A(x, y))=\frac{1}{a}\left[V^{\mathrm{I}}(x+y)+V(x+y)\left(\mathrm{E} \bar{X}^{*}+\frac{\mathrm{E} \xi^{2}}{2 a}\right)\right]+o(V(x+y))
$$

as $x \rightarrow \infty$.

Note that the coefficient $\mathrm{E} \bar{X}^{*}+\mathrm{E} \xi^{2} / 2 a$ in asymptotic expansion (45) depends on $\rho$ and is not uniformly bounded in $\rho \in\left(0, \rho_{0}\right)$, because $a^{*}=a_{\zeta}-r^{*} a_{\tau} \downarrow 0$ as $\rho \uparrow \rho_{0}\left(r^{*}=r-p(\rho)\right.$, $\rho_{0}$ is the solution to the equation $r-p(\rho)=a_{\zeta} / a_{\tau}$; see (17)). Hence, $\bar{X}^{*} \rightarrow \infty$ almost surely and $\mathrm{E} \bar{X}^{*} \rightarrow \infty$ as $\rho \uparrow \rho_{0}$.

On the other hand, for small $a^{*}$ (i.e. for $\rho$ close to $\rho_{0}$ ), we can use the results on transient phenomena to find the approximate (large) value of $\mathrm{E} \bar{X}^{*} \approx \operatorname{var}\left(\zeta-r^{*} \tau\right) / 2 a^{*}$ (see, e.g. [3]). Note also that the coefficient $\mathrm{E} \bar{X}^{*}+\mathrm{E} \xi^{2} / 2 a$ in the second term of asymptotic expansion (45) is always positive and, hence, the true values of $\mathrm{P}(A(x, y))$ for large $x$ always exceed their approximation $(1 / a) V^{\mathrm{I}}(x+y)$ given by Theorem 2 . 


\subsection{Proof of Theorem 7}

We will follow the same scheme of proof as for Theorem 2, but more precise estimates will be used.

The estimate for the summand $P_{2}$ in representation (26). The respective argument in the proof of Theorem 2 implies that

$$
\begin{aligned}
P_{2} & :=\int_{0}^{y(1-\varepsilon)} \mathrm{P}(\bar{X}>x, \chi(x) \in \mathrm{d} u) \mathrm{P}\left(\sup _{t \geq 0} X_{p(\cdot), u}(t)>y-u\right) \\
& \leq \mathrm{P}(\bar{X}>x) \mathrm{P}\left(\bar{X}^{*}>\varepsilon y\right) \\
& \leq c V^{I}(x) V^{I}(\varepsilon y), \quad c<\infty .
\end{aligned}
$$

The estimate for the summand $P_{3}$. Now consider the summand

$$
P_{3}:=\int_{y(1-\varepsilon)}^{y} \mathrm{P}(\eta(x)<\infty, \chi(x) \in \mathrm{d} u) \mathrm{P}\left(\sup _{t \geq 0} X_{p(\cdot), u}(t)>y-u\right) .
$$

We employ the integro-local theorem, Theorem 6, which implies that, for each fixed $\Delta>0$ and $u \in(y(1-\varepsilon), y)$, where $\varepsilon=o(1)$, we have

$$
\mathrm{P}(\eta(x)<\infty, \chi(x) \in \Delta(u]) \sim \frac{\Delta}{a} V(x+u) .
$$

Using these relations and standard analysis techniques, we can approximate the integral in (47) by

$$
\frac{1}{a} V(x+y) \int_{y(1-\varepsilon)}^{y} \mathrm{P}\left(\sup _{t \geq 0} X_{p(\cdot), u}(t)>y-u\right) \mathrm{d} u(1+o(1)) .
$$

This representation allows us to obtain an upper bound for $P_{3}$. Since $\varepsilon y \rightarrow \infty$ and the process $X_{p(\cdot), u}(t)$ is pathwise dominated in the range of values $(-\infty, y-u)$ by the process $X^{*}(t)$ (using a suitable construction of these processes on a common probability space), we have

$$
\begin{aligned}
P_{3} & \leq \frac{1}{a} V(x+y) \int_{0}^{\varepsilon y} \mathrm{P}\left(\bar{X}^{*}>z\right) \mathrm{d} z(1+o(1)) \\
& =\frac{1}{a} V(x+y) \mathrm{E} \bar{X}^{*}(1+o(1)), \quad \bar{X}^{*}=\sup _{t \geq 0} X^{*}(t) .
\end{aligned}
$$

(Here the finiteness of $\mathrm{E} \bar{X}^{*}$ follows from the finiteness of $\mathrm{E} \xi^{2}$.)

Now we obtain a lower bound for the integral in (48). We have

$$
\mathrm{P}\left(\sup _{t \geq 0} X_{p(\cdot), u}(t)>y-u\right) \geq \mathrm{P}\left(\sup _{t \geq 0} X_{p(\cdot), u}(t)>y-u ; \inf _{t \in(0, v(y-u))} X_{p(\cdot), u}(t)>-M r\right),
$$

where $v(y-u)$ is the time when the process $X_{p(\cdot), u}(t)$ first crosses the level $y-u$ and $M:=\sqrt{\varepsilon} y \rightarrow \infty$ as $y \rightarrow \infty$.

Denote by $X_{*}(t)$ the process defined in the same way as the process $X(t)$ but with the parameter $r$ changed to $r_{*}:=r-p(\rho(1-\sqrt{\varepsilon}))>a_{\zeta} / a_{\tau}$. The process $X_{p(\cdot), u}(t), u \in$ $(y(1-\varepsilon), y)$, is minorated in the range of values $(y-M, y)$ by the process $X_{*}(t)$ (we assume that these processes are constructed in a suitable way on a common probability space). The 
process $X_{*}(t)$ has a negative mean drift, as does $X(t)$. Therefore, if a trajectory of the process $X_{*}(t)$ reaches the level

$$
m:=y-u \in(0, \varepsilon y)
$$

not crossing the negative level $-M$ then the process $X_{p(\cdot), u}(t)$ surely reaches the level $m$ not crossing the level $-M$. Hence, if we set

$$
\nu_{*}(m):=\min \left\{t: X_{*}(t)>m\right\}
$$

then the probability

$$
\mathrm{P}\left(\sup _{t \geq 0} X_{*}(t)>m, \underline{X}\left(v_{*}(m)\right)>-M\right),
$$

where

$$
\underline{X}(v):=\inf _{t \leq v} X_{*}(t)
$$

does not exceed the right-hand side of (50). Thus (by (48) with $y-u$ changed to $m$ ),

$$
P_{3} \geq \frac{1}{a} V(x+y) \int_{0}^{\varepsilon y} \mathrm{P}\left(\bar{X}_{*}>m, \underline{X}\left(v_{*}(m)\right)>-M\right) \mathrm{d} m(1+o(1)),
$$

where $\bar{X}_{*}=\sup _{t \geq 0} X_{*}(t)$. To estimate this integral, we need the following lemma.

Lemma 1. For $m \leq \varepsilon y=o(M)(M=\sqrt{\varepsilon} y)$, the following relation holds:

$$
\mathrm{P}\left(\bar{X}_{*}>m, \underline{X}\left(v_{*}(m)\right)>-M\right) \sim \mathrm{P}\left(\bar{X}_{*}>m\right) .
$$

Proof. Since $\mathrm{P}\left(\bar{X}_{*}>m\right) \geq c_{1} V^{\mathrm{I}}(m), c_{1}=$ constant, it suffices to show that

$$
\mathrm{P}\left(\bar{X}_{*}>m, \underline{X}\left(v_{*}(m)\right) \leq-M\right)=o\left(V^{\mathrm{I}}(m)\right) .
$$

The event on the left-hand side of (52) means that the path of $X_{*}(t)$ first reaches the level $-M$ and then ascends to the level $m$. This means that the probability in (52) does not exceed

$$
\mathrm{P}\left(\bar{X}_{*}>M+m\right) \geq c_{2} V^{\mathrm{I}}(M+m)=o(V(m)), \quad c_{2}=\text { constant },
$$

because $m=o(m+M)$. This proves relation (52) and, hence, Lemma 1 .

Inequality (51) and Lemma 1 imply that

$$
P_{3} \geq \frac{1}{a} V(x+y) \int_{0}^{\varepsilon y} \mathrm{P}\left(\bar{X}_{*}>m\right) \mathrm{d} m(1+o(1))=\frac{\mathrm{E} \bar{X}_{*}}{a} V(x+y)(1+o(1)) .
$$

Now if we also prove that

$$
\mathrm{E} \bar{X}_{*} \rightarrow \mathrm{E} \bar{X}^{*}
$$

as $\varepsilon \rightarrow 0(y \rightarrow \infty, \varepsilon y \rightarrow \infty)$ then by (49) and (53) we will obtain

$$
P_{3} \sim \frac{\mathrm{E} \bar{X}^{*}}{a} V(x+y)(1+o(1)) .
$$

The required convergence (54) follows from the following statement. Slightly changing the already used notation, set

$$
\xi_{j}^{(r)}:=\zeta_{j}-r \tau_{j}, \quad X_{k}^{(r)}:=\sum_{j=1}^{k} \xi_{j}^{(r)}, \quad \bar{X}^{(r)}:=\sup _{k \geq 0} X_{k}^{(r)} .
$$

It is clear that the supremum of the corresponding generalized renewal process in continuous time coincides with $\bar{X}^{(r)}$. 
Lemma 2. Let $\mathrm{E} \xi=\mathrm{E}(\zeta-r \tau)<0$. Then the function $\mathrm{E} \bar{X}^{(u)}$ is continuous at the point $u=r$.

Proof. First we will prove left continuity, i.e. that

$$
\lim _{u \uparrow r} \mathrm{E} \bar{X}^{(u)}=\mathrm{E} \bar{X}^{(r)}
$$

For $u<r$, the trajectory $\left\{X_{k}^{(u)}\right\}_{k=1}^{\infty}$ dominates the trajectory $\left\{X_{k}^{(r)}\right\}_{k=1}^{\infty}$. The strong law of large numbers implies that $\bar{X}^{(r)}<\infty$ almost surely and that the supremum $\bar{X}^{(r)}$ is attained at a finite time interval. Therefore, $\bar{X}^{(u)} \downarrow \bar{X}^{(r)}$ as $u \uparrow r$. Thus, by the monotone convergence theorem, $\mathrm{E} \bar{X}^{(u)} \downarrow \mathrm{E} \bar{X}^{(r)}$ as $u \uparrow r$.

The right continuity of $\mathrm{E} \bar{X}^{(u)}$ is proved in exactly the same way. This completes the proof of the lemma.

Now note that Lemma 2 can be applied to the process $X^{*}(u)$ as well. Hence, relations (54) and (55) are proved.

The estimate for the summand $P_{1}$. We have

$$
P_{1}:=\mathrm{P}(\bar{X}>x, \chi(x)>y)=\mathrm{P}(\bar{X}>x+y)-P_{4}-P_{5},
$$

where

$$
\begin{aligned}
& P_{4}:=\int_{0}^{y(1-\varepsilon)} \mathrm{P}(\bar{X}>x, \chi(x) \in \mathrm{d} u) \mathrm{P}(\bar{X}>y-u), \\
& P_{5}:=\int_{y(1-\varepsilon)}^{y} \mathrm{P}(\bar{X}>x, \chi(x) \in \mathrm{d} u) \mathrm{P}(\bar{X}>y-u) .
\end{aligned}
$$

Here $\varepsilon>0$ tends to 0 as $x \rightarrow \infty$ in such a way that $\varepsilon y \rightarrow \infty$. But we have already estimated integrals of the same type as $P_{4}$ and $P_{5}$ (it was done under more complicated conditions). Proceeding similarly to proving (46) we obtain

$$
P_{4} \leq c V^{\mathrm{I}}(x) V^{\mathrm{I}}(\varepsilon y),
$$

and, similarly to (55),

$$
P_{5} \sim \frac{\mathrm{E} \bar{X}}{a} V(x+y) .
$$

Besides, Theorem 7.5.3 of [3] implies that, under condition $\left(\mathcal{R}_{2}\right)$, the following representation holds for nonlattice distributions of $\xi$ :

$$
\mathrm{P}(\bar{X}>x)=\frac{V^{\mathrm{I}}(x)}{a}+C V(x)+o(V(x)) \quad \text { as } x \rightarrow \infty,
$$

where

$$
C=\frac{\mathrm{E} \xi^{2}}{2 a^{2}}+\frac{\mathrm{E} \bar{X}}{a}
$$

Under condition $\left(\mathcal{R}_{3}\right)$, the distribution of $\xi$ is nonlattice, and, hence, we can apply relation (59) to (56), thus obtaining (see also (57) and (58))

$$
P_{1}=\frac{V^{\mathrm{I}}(x+y)}{a}+\left(C-\frac{\mathrm{E} \bar{X}}{a}\right) V(x+y)(1+o(1))+O\left(V^{\mathrm{I}}(x) V^{\mathrm{I}}(\varepsilon y)\right),
$$


where

$$
C-\frac{\mathrm{E} \bar{X}}{a}=\frac{\mathrm{E} \xi^{2}}{2 a^{2}} .
$$

Completing the proof of Theorem 7. It remains to estimate $V^{\mathrm{I}}(x) V^{\mathrm{I}}(\varepsilon y)$. Since

$$
V^{\mathrm{I}}(x) \sim \frac{x V(x)}{\alpha-1} \quad \text { and } \quad V(x)<x^{-\alpha+\beta}
$$

for each $\beta>0$ and all large enough $x$ (see, e.g. Theorem 1.1.3 of [3]), the following relation holds for $y=\rho x$ :

$$
V^{\mathrm{I}}(x) V^{\mathrm{I}}(\varepsilon y) \sim \rho^{\alpha-1} V^{\mathrm{I}}(x) V^{\mathrm{I}}(\varepsilon x)<\frac{\rho^{\alpha-1} x}{\alpha-1} V(x)(\varepsilon x)^{-\alpha+\beta+1} .
$$

In this relation, for $\beta<\alpha / 2-1$ and $\varepsilon \geq x^{(2-\alpha) / 2 \alpha}$, we have

$$
\begin{gathered}
x(\varepsilon x)^{-\alpha+\beta+1}<\varepsilon^{-\alpha / 2} x^{(2-\alpha) / 2} \leq x^{(2-\alpha) / 4} \rightarrow 0 \quad \text { as } x \rightarrow \infty, \\
V^{\mathrm{I}}(x) V^{\mathrm{I}}(\varepsilon y)=o(V(x)) \quad \text { as } \varepsilon \rightarrow 0 .
\end{gathered}
$$

Combining the above relations obtained for $P_{1}-P_{3}$ (see (46), (56), and (60)) and using (61), we obtain (45).

\section{References}

[1] Asmussen, S. (2007). Ruin Probabilities. World Scientific, River Edge, NJ.

[2] Asmussen, S. And Klüppelberg, C. (1996). Large deviations results for subexponential tails, with applications to insurance risk. Stoch. Process. Appl. 64, 103-125.

[3] Borovkov, A. A. And Borovkov, K. A. (2008). Asymptotic Analysis of Random Walks. Cambridge University Press.

[4] CAI, J. (2004). Ruin probabilities and penalty functions with stochastic rates of interest. Stoch. Process. Appl. 112, 53-78.

[5] CaI, J. (2007). On the time value of absolute ruin with debit interest. Adv. Appl. Prob. 39, 343-359.

[6] CAI, J. AND Dickson, D. C. M. (2002). On the expected discounted penalty function at ruin of a surplus process with interest. Insurance Math. Econom. 30, 389-404.

[7] Dassios, A. And Embrechts, P. (1989). Martingales and insurance risk. Stoch. Models 5, 181-217.

[8] Dickson, D. C. M. And Egídio dos Reis, A. D. (1997). The effect of interest on negative surplus. Insurance Math. Econom. 21, 1-16.

[9] Embrechts, P. ANd Schmidli, H. (1994). Ruin estimation for a general insurance risk model. Adv. Appl. Prob. 26, 404-422.

[10] Korshunov, D. A. (2001). Large deviation probabilities for maxima of sums of independent summands with negative mean and subexponential distribution. Theory Prob. Appl. 46, 355-365.

[11] Sundt, B. AND Teugels, J. L. (1995). Ruin estimates under interest force. Insurance Math. Econom. 16, 7-22. 